This article is licensed under the Creative Commons Attribution-NonCommercial 4.0 International License (CC BY-NC) (http://www.karger.com/Services/OpenAccessLicense). Usage and distribution for commercial purposes requires written permission.

\title{
Bullous Exudative Retinal Detachment after Retinal Pattern Scan Laser Photocoagulation in Diabetic Retinopathy
}

\author{
Shohei Nishikawa ${ }^{a}$ Hiroshi Kunikata ${ }^{a, b} \quad$ Naoko Aizawa $^{a}$ \\ Toru Nakazawa ${ }^{\mathrm{a}-\mathrm{d}}$ \\ aDepartment of Ophthalmology, Tohoku University Graduate School of Medicine, \\ Sendai, Japan; ${ }^{b}$ Department of Retinal Disease Control, Tohoku University Graduate \\ School of Medicine, Sendai, Japan; ${ }^{C}$ Department of Advanced Ophthalmic Medicine, \\ Tohoku University Graduate School of Medicine, Sendai, Japan; ${ }^{d}$ Department of \\ Ophthalmic Imaging and Information Analytics, Tohoku University Graduate School of \\ Medicine, Sendai, Japan
}

\section{Keywords}

Serous retinal detachment · Diabetic macular edema · Pattern scan laser · Complication . PASCAL
Abstract irreversible visual loss, even if the ERD resolves within 1 month.

Laser retinal photocoagulation is the gold standard treatment for diabetic retinopathy. We describe 3 cases in which bullous exudative retinal detachment (ERD) developed after pattern scan laser photocoagulation (PASCAL) in diabetic retinopathy. ERD spontaneously resolved in all 3 cases with various visual courses. This case series highlights 2 key points: first, ERD can occur regardless of gender, age, glycemic control, or vitreous status and despite a moderate number of laser shots, even with PASCAL; second, ERD in nonvitrectomized eyes may cause 


\section{Case Reports in Ophthalmology}

Case Rep Ophthalmol 2017;8:475-481

(c) 2017 The Author(s). Published by S. Karger AG, Basel www.karger.com/cop

Nishikawa et al.: Bullous Exudative Retinal Detachment after Retinal Pattern Scan Laser Photocoagulation in Diabetic Retinopathy

\section{Introduction}

Laser retinal photocoagulation is the gold standard treatment for diabetic retinopathy. This treatment partially destroys the outer retina, reducing oxygen demand and the production of inflammatory cytokines. This increases vitreous oxygenation and induces vasoconstriction. Recently, pattern scan laser photocoagulation (PASCAL) has been introduced, enabling short-pulse-duration retinal photocoagulation in many locations simultaneously [15]. However, a previous report on approximately 1,300 cases that underwent PASCAL showed that complications, although rare, were varied, including 17 cases of retinal bleeding $(1.3 \%), 2$ cases of choroidal detachment $(0.15 \%)$, and 1 case of exudative retinal detachment (ERD) $(0.07 \%)$ [2]. Though ERD was the rarest complication, it can severely impair visual function, and its characteristics and clinical course remain unclear. Here, we describe 3 cases in which bullous ERD developed after PASCAL in diabetic retinopathy. There have not been any other published reports on such an unusual case series.

\section{Case Report}

The first patient (case 1; Table 1; Fig. 1,2) was a 43-year-old man with untreated type 2 diabetes (HbA1c: 11\%). He was introduced to our department 11 months before this report and underwent intravitreal bevacizumab in both eyes, followed by pan-retinal laser photocoagulation. At the time of this report, the patient underwent additional treatment with 1,836 PASCAL laser shots for a remaining nonperfusion area (NPA) in his left eye (wave length: $532 \mathrm{~nm}$, diameter: $200 \mu \mathrm{m}$, duration: $20 \mathrm{~ms}$, power: $400 \sim 450 \mathrm{~mW}$ ). Three days after the treatment, ERD occurred and decimal visual acuity (VA) decreased from 0.4 to counting fingers. Unfortunately, though the ERD resolved spontaneously 1 month later, VA remained at 0.03 .

The second patient (case 2; Table 1; Fig. 1,2) was a 37-year-old woman with untreated type 2 diabetes (HbA1c: 14\%). She was introduced to our department 19 months before this report and underwent pan-retinal laser photocoagulation in both eyes. Her right eye had previously shown mild posterior synechia, and she had undergone cataract surgery and subsequent vitreous surgery with endolaser photocoagulation for vitreous hemorrhage 11 months before this report. At the time of this report, she underwent additional treatment with 1,056 PASCAL laser shots for a remaining NPA in her right eye (wave length: $532 \mathrm{~nm}$, diameter: $200 \mu \mathrm{m}$, duration: $20 \mathrm{~ms}$, power: $400 \sim 450 \mathrm{~mW}$ ). Two days after the treatment, ERD occurred and decimal VA decreased from 0.6 to 0.04 . Steroid treatment was considered but was not administered because of a quick (within 3 days) spontaneous reduction of the retinal detachment. Fortunately, the ERD resolved spontaneously 2 months later, and VA recovered to 0.8 .

The final patient (case 3; Table 1; Fig. 1, 2) was a 71-year-old man with untreated type 2 diabetes (HbA1c: 7.4\%). He was introduced to our department 4 months before this report, presenting with vitreous hemorrhage in his right eye. The right eye had undergone vitreous surgery 2 months previously. At the time of this study, the patient underwent additional treatment with 3,521 PASCAL laser shots for a remaining NPA in his right eye (wave length: $561 \mathrm{~nm}$, diameter: $200 \mu \mathrm{m}$, duration: $200 \mathrm{~ms}$, power: 270 400 mW). Three days after the 


\section{Case Reports in Ophthalmology}

Case Rep Ophthalmol 2017;8:475-481

(c) 2017 The Author(s). Published by S. Karger AG, Basel www.karger.com/cop

Nishikawa et al.: Bullous Exudative Retinal Detachment after Retinal Pattern Scan Laser Photocoagulation in Diabetic Retinopathy

treatment, ERD occurred and decimal VA decreased from 1.0 to 0.02 . Fortunately, the ERD resolved spontaneously 3 weeks later, and VA recovered to 0.6 .

All patients presented without any signs of inflammation, although mild posterior synechia was present in case 2 on the first visit. Furthermore, no patients had any history of uveitis or rheumatoid disorders.

\section{Discussion}

Though the reason why ERD occurred after photocoagulation remains unclear, ERD is generally thought to be caused by disruption of the blood-ocular barrier due to intraocular inflammation and endothelial capillary damage in the retina. Thus, blood-retinal barrier breakdown due to retinal pigment cell impairment after retinal PASCAL treatment is a possible cause of bullous ERD in this situation. ERD after photocoagulation is very rare, and, thus, its clinical features have not been elucidated [2]. This report is, therefore, the first to confirm, to some extent, details of the clinical course of ERD after photocoagulation and background characteristics related to the condition. Though ERD has been reported to occur in young male patients with poor glycemic control [2], the current report shows that ERD can occur regardless of gender, age, or glycemic control. Case 3 had relatively good glycemic control (HbA1c: 7.4\%) and underwent more than 3,000 PASCAL shots before ERD occurred. Thus, ERD may occur even after a moderate number of laser shots in patients with wellcontrolled diabetes. The clinical course of the present case series had a number of interesting aspects: ERD resolved spontaneously in all 3 cases; case 2 had good visual recovery despite having the longest duration of ERD ( 2.5 months); and cases 2 and 3 had good visual recovery under postoperative avitreous status before PASCAL. Case 1, with poor visual recovery, had no history of vitrectomy before PASCAL, suggesting that the vitreous gel may act as a reservoir of photocoagulation-induced inflammatory cytokines that cause irreversible retinal cell damage. Thus, this case series highlights 2 key points: first, ERD can occur regardless of gender, age, glycemic control, or vitreous status and despite a moderate number of laser shots, even with PASCAL; second, ERD in nonvitrectomized eyes may cause irreversible visual loss, even if the ERD resolves within 1 month.

\section{Statement of Ethics}

Informed consent for the treatment was obtained from the patients. The procedures conformed to the tenets of the Declaration of Helsinki, and the study was approved by the institutional review board of Tohoku University Graduate School of Medicine.

\section{Disclosure Statement}

None of the authors have any potential conflicts of interest to disclose. 
Nishikawa et al.: Bullous Exudative Retinal Detachment after Retinal Pattern Scan Laser Photocoagulation in Diabetic Retinopathy

\section{Funding Sources}

This paper was supported in part by a JST grant from JSPS KAKENHI Grants-in-Aid for Scientific Research (C) (H.K.: 26462629). The funders had no role in the design or conduct of the study; collection, management, analysis, and interpretation of the data; preparation, review, or approval of the manuscript; or the decision to submit the manuscript for publication.

\section{Author Contributions}

Data collection, management, and interpretation of the data (S.N., H.K., N.A., and T.N.), drafting of the manuscript (H.K.), and review or approval of the manuscript (H.K. and T.N.). The principal investigators, Dr. Shohei Nishikawa and Dr. Naoko Aizawa, had full access to all the data in the study and take responsibility for the integrity of the data and the accuracy of the data analysis.

\section{References}

1 Nagpal M, Marlecha S, Nagpal K: Comparison of laser photocoagulation for diabetic retinopathy using 532-nm standard laser versus multispot pattern scan laser. Retina 2010;30:452-458.

-2 Velez-Montoya R, Guerrero-Naranjo JL, Gonzalez-Mijares CC, et al: Pattern scan laser photocoagulation: safety and complications, experience after 1,301 consecutive cases. Br J Ophthalmol 2010;94:720-724.

-3 Ye H, Jin C, Zhong X, Tian Z, Lin W: Pattern scan laser photocoagulator on retinopathy: an annual clinical application summary. Eye Sci 2011;26:70-74.

-4 Muqit MM, Marcellino GR, Henson DB, et al: Optos-guided pattern scan laser (PASCAL)-targeted retinal photocoagulation in proliferative diabetic retinopathy. Acta Ophthalmol 2013;91:251-258.

-5 Seymenoglu RG, Ulusoy MO, Baser EF: Safety and efficacy of panretinal photocoagulation in patients with high-risk proliferative diabetic retinopathy using pattern scan laser versus conventional YAG laser. Kaohsiung J Med Sci 2016;32:22-26. 


\section{Case Reports in Ophthalmology}

Case Rep Ophthalmol 2017;8:475-481

(C) 2017 The Author(s). Published by S. Karger AG, Basel www.karger.com/cop

Nishikawa et al:: Bullous Exudative Retinal Detachment after Retinal Pattern Scan Laser

Photocoagulation in Diabetic Retinopathy
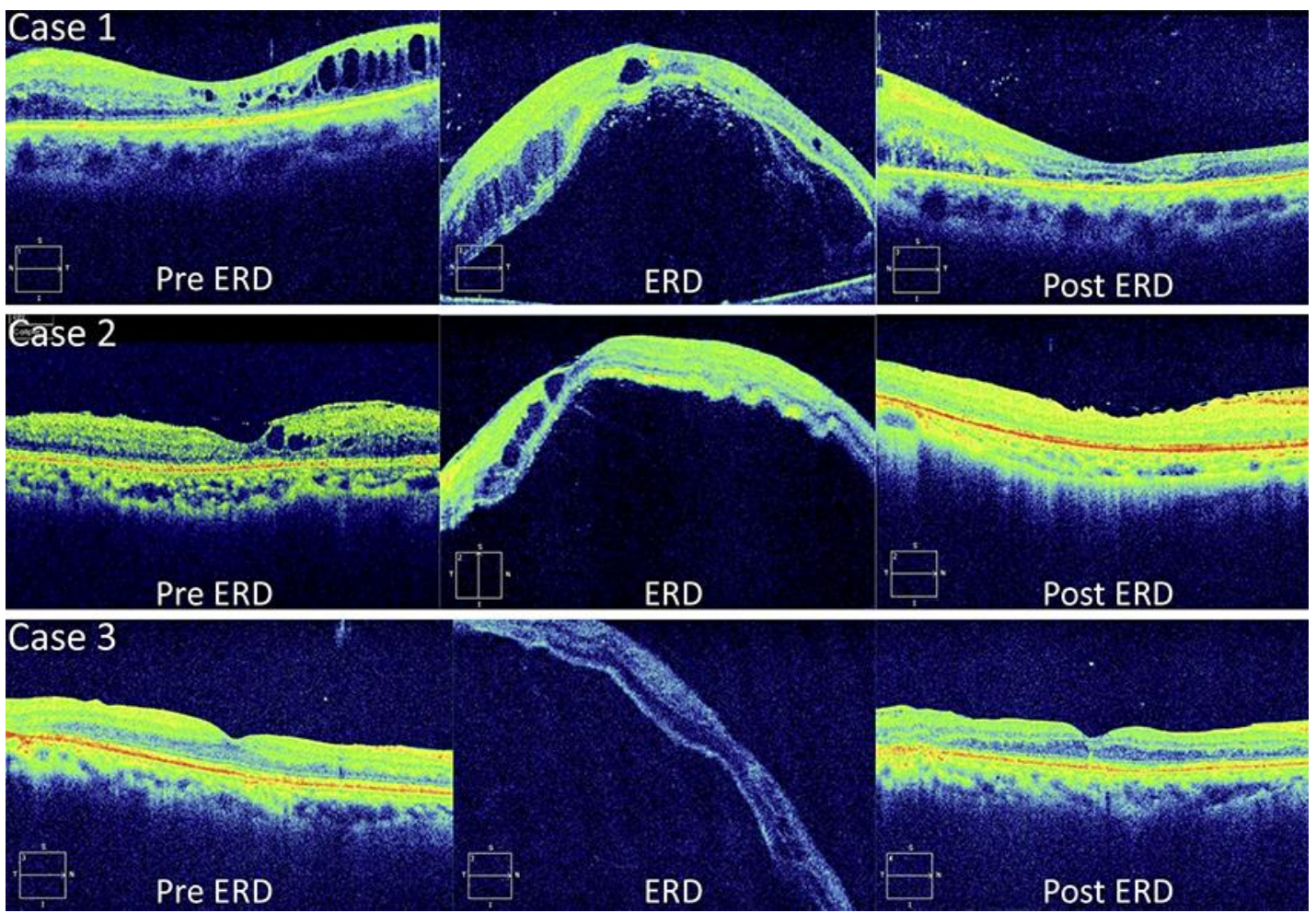

Fig. 1. Optical coherence tomography findings showing the time course of changes in 3 cases with exudative retinal detachment (ERD) after pattern scan laser photocoagulation (PASCAL). In the first patient (case 1, upper row), ERD occurred 3 days after PASCAL. Unfortunately, though the ERD resolved spontaneously 1 month later, visual function did not recover. In the second patient (case 2, middle row), ERD occurred 2 days after PASCAL. Fortunately, the ERD resolved spontaneously 2 months later, and visual function recovered successfully. In the final patient (case 3, lower row), ERD occurred 3 days after PASCAL. Fortunately, the ERD resolved spontaneously 3 weeks later, and visual function recovered moderately. 


\section{Case Reports in Ophthalmology}

Case Rep Ophthalmol 2017:8:475-481
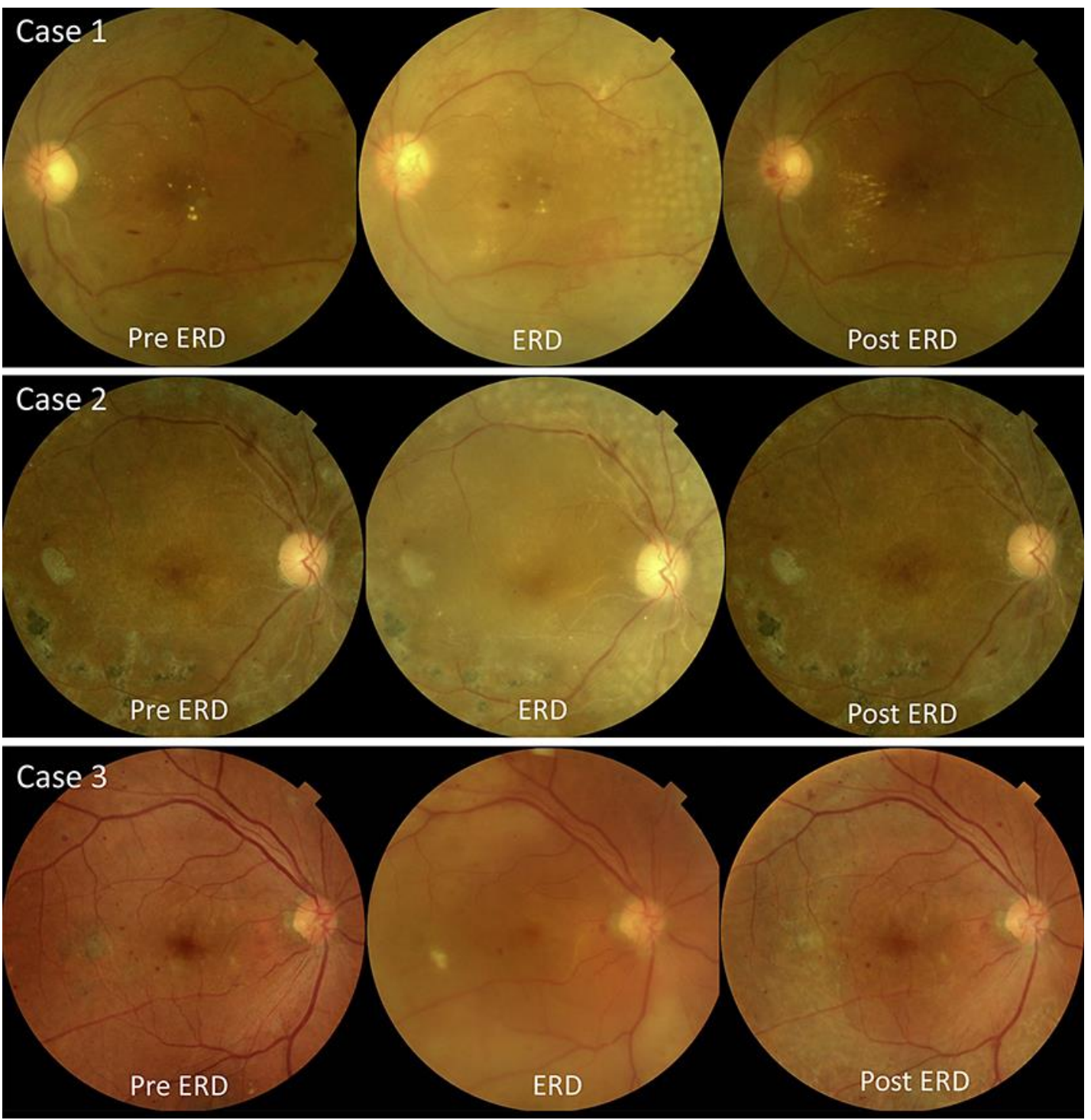

Fig. 2. Color fundus photographs showing the time course of changes in 3 cases with exudative retinal detachment (ERD) after pattern scan laser photocoagulation (PASCAL). In the first patient (case 1, upper row), ERD occurred 3 days after PASCAL. The ERD resolved spontaneously 1 month later, with poor visual recovery. In the second patient (case 2, middle row), ERD occurred 2 days after PASCAL. The ERD resolved spontaneously 2 months later, with good visual recovery. In the final patient (case 3, lower row), ERD occurred 3 days after PASCAL. The ERD resolved spontaneously 3 weeks later, with moderate visual recovery. 
Nishikawa et al.: Bullous Exudative Retinal Detachment after Retinal Pattern Scan Laser Photocoagulation in Diabetic Retinopathy

Table 1. Characteristics of eyes with exudative retinal detachment after retinal pattern scan laser photocoagulation in diabetic retinopathy

\begin{tabular}{|c|c|c|c|c|c|c|c|c|c|c|c|c|c|}
\hline \multirow[t]{2}{*}{ Case } & \multirow{2}{*}{$\begin{array}{l}\text { Age, } \\
\text { years }\end{array}$} & \multirow[t]{2}{*}{ Sex } & \multirow{2}{*}{$\begin{array}{l}\text { Diag- } \\
\text { nosis }\end{array}$} & \multirow{2}{*}{$\begin{array}{l}\mathrm{HbA} 1 \mathrm{c}, \\
\%\end{array}$} & \multirow{2}{*}{$\begin{array}{l}\text { Vitrectomy } \\
\text { history }\end{array}$} & \multicolumn{3}{|c|}{ Decimal BCVA } & \multicolumn{2}{|c|}{$\mathrm{FT}, \mu \mathrm{m}$} & \multirow{2}{*}{$\begin{array}{l}\text { PC spots, } \\
n\end{array}$} & \multirow{2}{*}{$\begin{array}{l}\text { Extent of } \\
\text { ERD, optic } \\
\text { disc area }\end{array}$} & \multirow{2}{*}{$\begin{array}{l}\text { Duration } \\
\text { of ERD, } \\
\text { days }\end{array}$} \\
\hline & & & & & & $\begin{array}{l}\text { pre } \\
\text { ERD }\end{array}$ & ERD & $\begin{array}{l}\text { post } \\
\text { ERD }\end{array}$ & $\begin{array}{l}\text { pre } \\
\text { ERD }\end{array}$ & $\begin{array}{l}\text { post } \\
\text { ERD }\end{array}$ & & & \\
\hline 1 & 43 & $\mathrm{~m}$ & PDR & 11.0 & - & 0.4 & $\mathrm{CF}$ & 0.03 & 207 & 183 & 1,836 & 3 & 31 \\
\hline 2 & 37 & $\mathrm{f}$ & PDR & 14.0 & + & 0.6 & 0.04 & 0.8 & 222 & 178 & 1,056 & total RD & 73 \\
\hline 3 & 71 & $\mathrm{~m}$ & PDR & 7.4 & + & 1.0 & 0.02 & 0.6 & 292 & 263 & 3,521 & 6 & 21 \\
\hline
\end{tabular}

BCVA, best-corrected visual acuity; FT, foveal thickness; ERD, exudative retinal detachment; PC, photocoagulation; PDR, proliferative diabetic retinopathy; $\mathrm{CF}$, counting fingers; $\mathrm{RD}$, retinal detachment. 